\title{
Impacto de um programa de competências parentais no stress e competências de atenção plena
}

Effects of a parenting education program on parenting skills, parenting stress, and mindfulness skills Impacto de un programa de competencias parentales en el estrés y en competencias de atención plena

\author{
Ana Isabel Pratas Martins dos Santos ${ }^{1}$ id hitps:/orcid.org/0000-0002-4816-9265 \\ Emília da Conceição Figueiredo Martins² ${ }^{2}$ h https://orcid.org/0000-0001-8697-0195 \\ Cátia Clara Ávila Magalhães ${ }^{2}$ io https://orcid.org/0000-0001-8018-4249 \\ Francisco Emiliano Dias Mendes² io https://orcid.org/0000-0002-7354-133X \\ Rosina Inês Ribeiro de Sá Fernandes² io https://orcid.orgr/0000-0002-6630-9831
} Santos Al, Martins EC, Magalhães CC, Mendes $\mathrm{FE}$, Fernandes RI. Impacto de um programa de competências parentais no stress e competências de atenção plena. Acta Paul Enferm. 2020;33:eAPE20190282.

DOI http://dx.doi.org/10.37689/actaape/2020AE02826

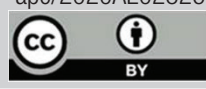
Poder familiar; Atenção plena; Educação infantil

Keywords Education, nonprofessional; Parental education; Parenting; Mindfulness; Child rearing

Descriptores

Educación no professional: Educación parental; Responsabilidad parental; Atención plena; Crianza del niño

Submetido 5 de Outubro de 2019

Aceito

24 de Março de 2020

\section{Autor correspondente}

Cátia Magalhães

E-mail: cmagalhaes@esev.ipv.pt

\section{Resumo}

Objetivo: Analisar o impacto da frequência do Adults and Children Together Raising Safe Kids (ACT-RSK) Programe nas competências parentais, no stress parental e nas competências de atenção plena, de um grupo de pais de crianças.

Métodos: Realizou-se uma investigação-ação, utilizando um pré e pós-teste, tendo por referência a implementação do programa ACT-RSK, em 8 sessões de 90 minutos com periodicidade semanal, numa amostra de 22 pais da região Centro de Portugal. Utilizaram-se os questionários do Programa ACT-RSK, o Índice de Stress Parental, a Escala de Mindfulness Interpessoal na Parentalidade e um questionário sociodemográfico. A análise descritiva e inferencial (Wilcoxon) foi realizada com recurso ao Statistical Package fos Social Sciences (SPSS - IBM 24), com grau de confiança de 95\%.

Resultados: Os participantes melhoraram, do pré para pós-teste, nas 4 dimensões do programa: estilos parentais $(M=36.32 \pm 6.61$ vs. $M=48.50 \pm 4.31 ; p<0.01)$; meios de comunicação $(M=24.73 \pm 3.90$ vs. $M=30.73 \pm 2.90 ; p \leq 0.01)$; desenvolvimento infantil $(M=55.18 \pm 11.82$ vs. $M=73.55 \pm 4.04 ; p \leq 0.01)$; e comportamento dos pais ( $M=34.23 \pm 7.83$ vs. $M=43.05 \pm 2.81 ; p \leq 0.01$ ). Destacam-se, igualmente, melhorias consideráveis nos resultados da Escala de Mindfulness Interpessoal na Parentalidade $(Z=-3.929, p=0.000)$ e no stress parental, que desceu do nível muito elevado (91.59) para o nível médio (74.45).

Conclusão: Constata-se o impacto positivo do ACT-RSK na melhoria das variáveis estudadas.

\section{Abstract}

Objective: To analyze the impact of the Adults and Children Together Raising Safe Kids (ACT-RSK) Program in parenting skills, parenting stress, and mindfulness skills in a group of parents and children.

Methods: This investigative intervention was based on the application of a pre-test and a post-test before and after the implementation of the ACT-RSK Program. The program consisted of eight weekly 90-minute sessions conducted with a sample of 22 parents from Central Portugal. The ACT-RSK Program questionnaires, Parenting Stress Index (PSI), Interpersonal Mindfulness in Parenting (IM-P) scale, and a socio-demographic questionnaire were used. A descriptive and inferential analysis (Wilcoxon) was conducted using the Statistical Package for Social Sciences (BBM SPSS, v. 24) with a 95\% confidence interval.

Results: Participants showed improvement from pre-test to post-test in all four program dimensions: Parenting Style $(M=36.32 \pm 6.61$ vs. $M=48.50 \pm 4.31 ; p<0.01)$; Electronic Media $(M=24.73 \pm 3.90$ vs. $M=30.73 \pm 2.90 ; p \leq 0.01)$; Child Development $(M=55.18 \pm 11.82$ vs. $M=73.55 \pm 4.04 ; p \leq 0.01)$; and Parental Behavior ( $M=34.23 \pm 7.83$ vs. $M=43.05 \pm 2.81 ; p \leq 0.01$ ). Likewise, significant improvement was seen in the Interpersonal Mindfulness in Parenting (IM-P) scale's results $(Z=-3.929, p=0.000)$ and in parenting stress, which decreased from a very high (91.59) to a medium level (74.45). 
Conclusion: Data show the ACT-RSK had a positive effect and improved study variables.

\section{Resumen}

Objetivo: Analizar el impacto de la frecuencia del Adults and Children Together Raising Safe Kids (ACT-RSK) Programe en las competencias parentales, en el estrés parental y en las competencias de atención plena de un grupo de padres de niños.

Métodos: Se realizó una investigación-acción utilizando un pretest y postest en la implementación del programa ACT-RSK, en 8 sesiones de 90 minutos con frecuencia semanal, en una muestra de 22 padres de la región Centro de Portugal. Se utilizaron los cuestionarios del programa ACT-RSK, el Índice de Estrés Parental, la Escala de Mindfulness Interpersonal en la Paternidad y un cuestionario sociodemográfico. El análisis descriptivo e inferencial (Wilcoxon) fue realizado con el Statistical Package for Social Sciences (SPSS - IBM 24), con nivel de confianza de 95 \%.

Resultados: Del pretest al postest, los participantes demostraron una mejora en las cuatro dimensiones del programa: estilos parentales ( $(M=36,32 \pm 6,61$ vs. $M=48,50 \pm 4,31 ; p<0,01)$; medios de comunicación ( $M=24,73 \pm 3,90$ vs. $M=30,73 \pm 2,90 ; p \leq 0,01)$; desarrollo infantil $(M=55,18 \pm 11,82$ vs. $M=73,55 \pm 4,04$; $p \leq 0,01)$; y comportamiento de los padres ( $M=34,23 \pm 7,83$ vs. $M=43,05 \pm 2,81 ; p \leq 0,01)$. Además, se destacan mejoras considerables en los resultados de la Escala de Mindfulness Interpersonal en la Paternidad $(Z=-3,929, p=0,000)$ y el en estrés parental, que se redujo de un nivel muy elevado $(91,59)$ a un nivel medio $(74,45)$.

Conclusión: Se verifica un impacto positivo del ACT-RSK en la mejora de las variables estudiadas.

\section{Introdução}

Desde tempos imemoriáveis que dúvidas não restam sobre o papel fundamental da família, e dos pais em particular, na educação e desenvolvimento dos seus filhos. Todavia, tal papel reclama um conjunto de competências parentais que não são inatas no Homem e que, não raras vezes, são praticamente inexistentes em algumas famílias. É aliás nesse sentido que a Convenção sobre os Direitos da Criança, ${ }^{(1)}$ assim como a Constituição da República Portuguesa ${ }^{(2)}$ admitem a possibilidade da criança ser afastada dos seus pais se o seu superior interesse for colocado em crise.

A família desempenha um papel socializante fundamental na aprendizagem e processo de desenvolvimento social, constituindo-se como um modelo para muitos tipos de comportamentos e atitudes. ${ }^{(3)}$ Apresenta-se como o fator de proteção mais importante, contudo, nem sempre os pais conseguem agilizar competências e recursos emocionais para lidar com a exigência que o exercício de práticas educativas parentais pressupóe. Deste modo, afeta a capacidade parental para avaliar as necessidades da criança e fornecer-lhe as respostas adequadas, perspetivando-se assim, como um fator capaz de incitar práticas educativas parentais disfuncionais com repercussóes no bem-estar de pais e crianças, podendo potenciar o desenvolvimento de problemas emocionais e comportamentais nos filhos. ${ }^{(4)}$ Aliás, Kumpfer e Magalhães ${ }^{(5)}$ salientam que o foco na melhoria dos mecanismos familiares de proteção se traduz num aumento das competências parentais, favorecendo a organização familiar e reforçando as forças e proces- sos de resiliência, o que potencia um aumento dos recursos disponíveis e uma melhoria nas relações.

Neste contexto, a evidência científica sublinha a importância de práticas parentais baseadas na atenção plena, enquanto tomada de consciência e reflexão sobre os padróes pessoais na relação com os filhos, de modo a potenciar mudanças positivas. Estas apresentam-se como um conjunto de habilidades/competências que validam a qualidade das relaçóes entre pais e filhos, promovendo cuidados responsivos e sensíveis às necessidades da criança, podendo reduzir o nível de stress familiar, facilitando o estabelecimento de relaçóes seguras. ${ }^{(6)}$ Os programas de educação parental baseados em evidência, centram-se na promoção e no fortalecimento das famílias, apoiam os pais na mudança do seu comportamento parental e na perceção dos seus sentimentos e expetativas, conduzindo a modificaçóes no relacionamento com os seus filhos. (7) Porém, em Portugal como no Brasil, as intervençôes sistemáticas e baseadas em evidência que visam promover as práticas educativas parentais positivas são, ainda, muito escassas, havendo espaço para um longo caminho a percorrer. ${ }^{(8)}$

A relevância das práticas educativas parentais atinge proporçóes tais, que requer a mobilização de técnicos de diversas áreas para intervir junto de pais, sobretudo quando o objetivo principal é a prevenção, mas também na remediação. Entre estes profissionais, destacamos os das áreas socioeducativa e da saúde. Nestes últimos, são mediadores privilegiados os técnicos de saúde pública e comunitária, sobretudo os enfermeiros que lidam mais diretamente com as famílias e populaçóes. A literatura tem vindo a reforçar esta evidência, como é o caso de uma meta-análise que destaca o impacto duradouro e 
positivo de uma intervenção parental implementada em contexto hospitalar ${ }^{(9)}$ e também de revisóes sistemáticas que mostram resultados muito favoráveis de intervençóes de enfermeiros na capacitação parental nas relaçóes dos pais com crianças até aos seis anos. ${ }^{(10-12)}$ Neste contexto, os programas estruturados e com base na evidência tornam-se uma ferramenta essencial, em cuja decisão importam aspetos relacionados com o custo-benefício, sendo os que implicam intervenção em contexto familiar muito mais onerosos, ${ }^{(13)}$ pelo que o ACT-RSK se pode constituir como extremamente vantajoso. Com efeito, trata-se de um programa que exige formação dos técnicos, mas consiste em sessóes de grupo junto de pais, não implicando visitas domiciliárias. Destacamos, ainda, a popularidade em contexto de saúde, dos programas de preparaçáo o exercício de práticas educativas parentais que incluem a abordagem às técnicas de atenção plena. ${ }^{(14)}$

No presente estudo, pela sua relevância e evidência científica, optou-se por selecionar o Programa de Educação Parental - Educar Crianças em Ambiente Seguros (ACT-RSK), desenvolvido pela American Psychological Association (APA), ${ }^{(15)}$ que visa ajudar pais e cuidadores a criar filhos em ambientes saudáveis e sem violência. Tem como objetivo principal prevenir, reduzir ou eliminar a violência e os maus-tratos de crianças, na comunidade em geral e em contextos de vulnerabilidade familiar e social, promovendo a mudança dos comportamentos parentais e da relação pais-filhos, bem como a melhoria do comportamento infantil. Baseia-se numa abordagem psicoeducativa e cognitivo-comportamental e suporta-se nos seguintes princípios: a violência resulta da falta de habilidades de resolução de problemas e sociais necessárias para lidar com conflitos; as crianças aprendem por meio da observação e imitação; os adultos podem aprender a serem modelos e ensinar habilidades sociais às crianças, o que as ajudará a lidarem com as suas relaçôes sociais de forma não agressiva. ${ }^{(16,17)}$

Considerando a importância da primeira infância, o ACT-RSK arroga-se um programa promissor de intervenção parental preventiva. ${ }^{(3,17)}$ Encontra-se numa fase de adaptação à realidade portuguesa, havendo ainda, em Portugal, poucos estudos sobre a sua implementação e impacto nas autoperceçóes dos pais sobre os estilos e práticas parentais. Todavia, alguns trabalhos têm revelado resultados positivos e a concomitan- te validade do programa, sobretudo em contextos de teses acadêmicas. ${ }^{(18)}$ No Brasil, destacamos um estudo experimental, que avaliou a eficácia preventiva nas práticas educativas parentais do programa ACT-RSK e demonstrou, independentemente do nível socioeconómico ou tipo de escola, uma melhoria das práticas parentais e das estratégias educacionais positivas, bem como impacto positivo na diminuição ou eliminação da violência e maus tratos, para além da aferição da validade do programa no contexto brasileiro. ${ }^{(17)}$

Com efeito, os maus-tratos infantis são um problema substancial em todas as sociedades, inclusive nos países desenvolvidos e nos países em desenvolvimento, sendo realidade preocupante também em cenário brasileiro. ${ }^{(19)}$ Em muitos casos, o uso de práticas de disciplina violentas pode ser o resultado da raiva e frustração ou falta de conhecimento de práticas parentais não violentas. ${ }^{(20)} \mathrm{O}$ presente estudo poderá contribuir para o incremento da implementação do programa ACT-RSK na comunidade em geral e em contextos de vulnerabilidade familiar e social, promovendo a mudança dos comportamentos parentais e da relação pais-filhos e assumindo um papel essencial na prevenção do mau trato infantil.

Formulámos o seguinte problema de estudo: quais as alteraçóes verificadas nas competências parentais de um grupo de pais de crianças entre os 3 e os 9 anos de idade, da região Centro de Portugal, após participar no Programa ACT-RSK e como se relacionam as competências parentais e stress parental? Neste sentido, procurou-se analisar o impacto do Programa ACT-RSK nas competências parentais, no stress parental e nas competências de atenção plena, de um grupo de pais de crianças. Considerou-se a variável stress parental, pelas evidências na literatura sobre a sua importância nas práticas educativas parentais. ${ }^{(5,6)}$

\section{Métodos}

Trata-se de um estudo de natureza quantitativa, de investigação-ação, cujo desenho se norteou pela aplicação de pré e pós-teste, tendo por referência a implementação do programa ACT-RSK.

A amostra, constituída por 22 pais da região Centro de Portugal, foi selecionada por conveniência, através da 
sua sensibilização para participar no programa, efetuada por professores e educadores de infância, que se dispuseram a colaborar com os autores. Entre os participantes, que aceitaram colaborar de modo voluntário, 95.5\% são femininos, $37.1 \%$ com habilitação superior e as idades variam entre os 25 e os 48 anos $(M=38.18 \pm 5.42)$. Foram garantidos os procedimentos de natureza ética de acordo com a Declaração de Helsínquia.

As 8 sessóes que constituem o programa, com 90 minutos cada, foram implementadas com periodicidade semanal, nos 22 pais, divididos em três grupos de 6 , 8 e 8 participantes, em salas cedidas por duas instituiçôes escolares e por um centro local de desenvolvimento social, devidamente preparadas para o efeito (equipadas com quadro interativo, recurso importante para apresentação dos conteúdos e visionamento de filmes). A dinamizadora das sessões frequentou a formação obrigatória para facilitadores do programa ACT-RSK, com respetivo acompanhamento e supervisão de um International Master Trainer certificado pela APA.

Uma vez que os participantes já apresentavam indicadores positivos de práticas parentais, decorrentes da avaliação pré-teste, a participação no programa aconteceu numa lógica de otimização das suas competências.

Utilizou-se o questionário do Programa ACTRSK que pretendeu avaliar as competências parentais relativamente a quatro dimensóes: estilo parental; meios eletrónicos; desenvolvimento infantil; comportamento dos pais. Para avaliar o stress parental, recorreu-se à adaptação portuguesa da versão reduzida do Parenting Stress Index (PSI) ${ }^{(19,21)}$ denominada Índice de Stress Parental. O instrumento faculta um resultado global (indicativo do nível de stress experimentado pela figura parental) e resultados parciais em cada uma das três subescalas criança (criança difícil, dificuldade parental, interação disfuncional mãe/pai-criança). A escala é constituída por 36 itens, variando a pontuaçáo de cada subescala entre $12 \mathrm{e}$ 60 pontos. Resultados mais elevados correspondem a níveis mais altos de stress parental. ${ }^{(20)}$ Para avaliar as competências de atenção plena nas práticas educativas parentais, recorreu-se à Escala de Mindfulness Interpessoal na Parentalidade (EMIP). O instrumento avalia competências de atenção plena no contexto parental de acordo com 6 dimensóes: (1) escutar com atenção plena; (2) consciência emocional do self; (3) consciência emocional da criança;(4) autorregulação na relação parental; (5) não julgamento e aceitação do papel parental; (6) compaixão pela criança. ${ }^{(5)}$ Foi também aplicado um questionário sociodemográfico e de caracterização sociofamiliar.

A análise de dados afetuou-se através do SPSS (versão 24.0), com recurso a técnicas estatísticas descritivas (média, desvio padrão, mínimo e máximo) e inferenciais ajustadas à natureza dos dados e características da amostra, optando-se por testes não paramétricos (Wilcoxon). O grau de confiança foi de 95\%, o mais comummente utilizado em Ciências Sociais.

\section{Resultados}

Constatam-se valores médios superiores nas quatro dimensôes do programa ACT-RSK (desenvolvimento infantil; estilos parentais; meios de comunicação; comportamento dos pais) no pós-teste, ou seja, após a implementação do programa (Tabela 1). Para os pais, a participação nas sessôes do ACT-RSK, traduziu-se numa melhoria dos conhecimentos e crenças sobre a prevenção da violência, assim como nos comportamentos relacionados com as práticas educativas parentais. Pese embora os participantes não se encontrarem numa situação de risco sinalizada na relação parental, é possível indicar uma trajetória positiva no decurso deste programa.

Tabela 1. Média e desvio padrão nas quatro dimensões do ACT-RSK no pré e pós-teste

\begin{tabular}{lcccccc}
\hline \multirow{2}{*}{ Subescalas } & \multirow{2}{*}{$N^{*}$ itens } & \multicolumn{2}{c}{ Pré-teste } & Pós-teste & Pré-teste & Pós-teste \\
& \multicolumn{2}{c}{ M } & \multicolumn{2}{c}{ DP } \\
\hline Estilos parentais & 11 & 36,32 & 48.50 & 6.61 & 4.31 \\
Meios de comunicação & 9 & 24,73 & 30.73 & 3.90 & 2.90 \\
Desenvolvimento infantil & 12 & 55,18 & 73.55 & 11.82 & 4.04 \\
Comportamento dos pais & 10 & 34,23 & 43.05 & 7.83 & 2.81 \\
Score global & 42 & 150,45 & 195.82 & 26.01 & 10.90 \\
\hline
\end{tabular}

Analisando os valores médios (Tabela 2) e tomando o valor 90 como ponto de corte para níveis altos de stress, constata-se, nos momentos de pré e pós-teste, níveis muito elevado (91.59) e médio (74.45) de stress, respetivamente.

A média da escala total de atenção plena nas práticas educativas parentais foi de $102.91 \pm 12.07$, no pré-teste 
Tabela 2. Média, valores mínimos, máximos e desvio padrão das subescalas do índice de stress parental no pré e pós-teste

\begin{tabular}{lcccccccccc}
\hline Subescalas & $\begin{array}{c}\text { No } \\
\text { Itens }\end{array}$ & $\begin{array}{c}\text { Pré- } \\
\text { teste }\end{array}$ & $\begin{array}{c}\text { Pós- } \\
\text { teste }\end{array}$ & \multicolumn{2}{c}{ Pré-teste } & \multicolumn{2}{c}{ Pós-teste } & \multicolumn{2}{c}{$\begin{array}{c}\text { Pré- } \\
\text { teste }\end{array}$} & $\begin{array}{c}\text { Pós- } \\
\text { teste } \\
\text { DP }\end{array}$ \\
\hline Pais & 12 & 31,36 & 22,95 & 13 & 52 & 12 & 34 & 9,59 & 6,31 \\
Interação & 12 & 24,68 & 19,95 & 13 & 44 & 12 & 30 & 8,74 & 5,37 \\
Criança & 12 & 35,55 & 31,55 & 29 & 47 & 24 & 39 & 5,78 & 4,37 \\
Score global & 36 & 91,59 & 74,45 & 55 & 143 & 48 & 103 & 21,46 & 13,11 \\
\hline
\end{tabular}

M - média, DP - Desvio padrão

e 120.41 77.68, no pós-teste (Tabela 3), verificando-se valores médios mais elevados em todas as subescalas.

Tabela 3. Média e desvio padrão das subescalas da atenção plena nas práticas educativas parentais no pré e pós-teste

\begin{tabular}{|c|c|c|c|c|c|}
\hline \multirow[t]{2}{*}{ Subescalas } & \multirow{2}{*}{$\begin{array}{l}\mathrm{N}^{\circ} \\
\text { itens }\end{array}$} & $\begin{array}{l}\text { Pré- } \\
\text { teste }\end{array}$ & $\begin{array}{l}\text { Pós- } \\
\text { teste }\end{array}$ & $\begin{array}{l}\text { Pré- } \\
\text { teste }\end{array}$ & $\begin{array}{l}\text { Pós- } \\
\text { teste }\end{array}$ \\
\hline & & \multicolumn{2}{|c|}{ M } & \multicolumn{2}{|c|}{ DP } \\
\hline Escutar com atenção plena & 5 & 16,77 & 18,63 & 2,35 & 1,53 \\
\hline Compaixão pela criança & 6 & 23,95 & 27,05 & 3,18 & 2,38 \\
\hline Autorregulação na relação parental & 8 & 23,73 & 29,45 & 3,67 & 2,63 \\
\hline $\begin{array}{l}\text { Não julgamento e aceitação do } \\
\text { papel parental }\end{array}$ & 7 & 20,40 & 24,78 & 4,40 & 2,58 \\
\hline Consciência emocional da criança & 3 & 10,73 & 12,95 & 2,00 & 1,59 \\
\hline Consciência emocional de si & 2 & 7,32 & 7,55 & 1,25 & 1,65 \\
\hline Score global & 31 & 102,91 & 120,41 & 12,07 & 7,68 \\
\hline
\end{tabular}

M - média, DP - Desvio padrão

No que concerne à análise inferencial e considerando o resultado do teste Wilcoxon, registam-se alteraçóes estatisticamente significativas em todas as subescalas e no score global, das competências parentais do programa, representando melhorias consideráveis (Tabela 4).

Tabela 4. Resultados do Wilcoxon no pré e pós-teste das competências parentais do ACT-RSK

\begin{tabular}{lcc}
\hline Dimensões & Z & p-value \\
\hline Estilos parentais & -3.800 & 0.000 \\
Meios de comunicação & -3.828 & 0.000 \\
Comportamento dos pais & -3.931 & 0.000 \\
Desenvolvimento infantil & -4.110 & 0.000 \\
Desenvolvimento infantil (História 1) & $-3,559$ & 0.000 \\
Desenvolvimento infantil (História 2) & -4.019 & 0.000 \\
Desenvolvimento infantil (História 3) & -4.025 & 0.000 \\
Desenvolvimento infantil (História 4) & -3.450 & 0.001 \\
Score global & -4.108 & 0.000 \\
\hline
\end{tabular}

Z - Wilcoxon; p - nivel de significância

Igualmente, no score global e em todas as subescalas da atenção plena nas práticas educativas parentais, à exceção da Consciência emocional de si, se registam alteraçôes estatisticamente significativas (Tabela 5). O Programa ACT-RSK também parece evidenciar um efeito positivo no desenvolvimento destas competências.
Tabela 5. Resultados do Wilcoxon, no pré e pós-teste, de competências de atenção plena no contexto parental

\begin{tabular}{lcc}
\hline Dimensões & Z & p-value \\
\hline Escutar com atenção plena & -2.497 & 0.013 \\
Compaixão pela criança & -3.396 & 0.001 \\
Autorregulação na relação parental & -3.989 & 0.000 \\
Não julgamento e aceitação do papel parental & -3.447 & 0.001 \\
Consciência emocional da criança & -3.115 & 0.002 \\
Consciência emocional de si & -0.365 & 0.715 \\
Score global & -3.929 & 0.000 \\
\hline Z-Wilcoxon; p-nível de significância & &
\end{tabular}

\section{Discussão}

De acordo com os resultados obtidos, a intervenção com o programa ACT-RSK é eficaz para melhorar as práticas parentais. No que se refere à perceçáo dos participantes, o ACT-RSK ajudou-os a tornarem-se melhores pais, a compreender o comportamento da criança tendo em conta a sua fase de desenvolvimento, a lidar e controlar a raiva, a compreender o impacto dos meios de comunicação e a saber reduzir a exposição das crianças, bem como a utilizar práticas parentais mais positivas e eficazes. Estas mudanças permitiram percecionar uma melhoria no comportamento dos seus filhos. Também outros estudos $^{(3,17,22-26)}$ demostraram a eficácia do ACTRSK na melhoria das práticas parentais positivas e do comportamento da criança. É o caso dos resultados obtidos por Leça, ${ }^{(24)} \mathrm{Com}$ um grupo de 11 pais de crianças portuguesas em idade pré escolar, que reconheceram o impacto positivo da participação no programa ACTRSK, através de mudanças centradas em si próprios, nas crianças e na família. As principais mudanças situaram-se ao nível da autorregulação/reatividade emocional (sobretudo mais calma na gestão de conlfitos), na dimensão cognitiva (maior conhecimento sobre a criança, capacidade de reflexão e também atenção aos meios eletrónicos de comunicação) e na dimensão comportamental (aumento de práticas positivas e diminuição de práticas negativas). Igualmente Oliveira ${ }^{(25)}$ estudou a efetividade do Programa em 30 mães brasileiras de crianças entre um e sete anos, em condição de vulnerabilidade, todas nascidas de pré-termo. A comparação entre avaliaçóes pré e pós intervenção (Wilcoxon) revelou melhoria nos fatores de comunicação e regulação emocional e comportamental, na consciência parental, encorajamento positivo e ajustamento parental, bem como diminuição do uso de práticas coercivas. Também o comportamento das crianças, quer em termos de perceção das mães, 
quer em termos de sintomas emocionais, problemas de conduta e de relacionamento com os colegas, teve uma evolução positiva. Com efeito, na perceção das mães, o ACT-RSK apresentou-se com efetividade na modificação de práticas parentais e redução de dificuldades de comportamento nas crianças, evidenciando potencial como fator protetor do desenvolvimento de crianças na condição de vulnerabilidade. Também um estudo de caso $^{(26)}$ efetuado no Brasil (S. Paulo), com uma mãe de 36 anos de uma criança de 6 anos de idade, de elevado nível socioeconómico e académico, destacou que o Programa ACT foi autopercecionado pela mãe como benéfico, servindo para aprimorar o seu estilo parental, através da melhoria de conhecimentos sobre práticas parentais positivas. Este trabalho também realçou a importância do Programa em pais com nível socioeconómico elevado, o que foi corroborado com o grupo de pais da nossa investigaçáo e por outros autores. ${ }^{(18,20)}$

Os resultados sugerem, ainda, mudanças positivas no que respeita à aquisição/otimização de conhecimentos em todas as dimensóes do programa, refletindo-se positivamente no score global, corroborando um estudo anterior de Knox, Burkhart e Howe, ${ }^{(27)}$ em que pais e cuidadores demonstraram melhorias ao nível do conhecimento, comportamentos e crenças em relação à prevenção da violência e práticas educativas parentais, com a frequência do ACT-RSK. Knox e Burkhart ${ }^{(16)}$ comprovaram, também, um aumento do comportamento carinhoso dos pais, a diminuição das práticas educativas parentais severas e da disciplina negativa, bem como a diminuiçáo dos problemas de comportamento infantil, após a conclusão do programa ACT-RSK.

Acrescem melhorias ao nível do stress parental nos pais que se submeteram ao Programa ACT-RSK no nosso estudo. Um trabalho recente, ${ }^{(28)}$ mas ainda em desenvolvimento, com a implementação do Programa em mães de crianças entre 30 e 42 meses de idade na cidade brasileira de Pelotas, com recurso a um estudo randomizado controlado, com pré (1-3 meses) e pós teste (4-6 meses) e um follow-up (9-13 meses), pretende avaliar o impacto do programa na redução de práticas educativas parentais severas e de fatores de risco para a violência, onde se inclui o stress parental. Aguardamos resultados, porque os autores ainda só publicaram a primeira versão do protocolo do estudo. Porém, as expetativas da importância des- te contributo fundam-se no delineamento do estudo (ensaio clínico randomizado), não utilizado em qualquer outro de que tivemos conhecimento.

Em concordância com os resultados que obtivemos, a evidência demonstra que ao disponibilizar-se à família formas alternativas, eficientes e positivas, para lidar com os comportamentos dos filhos, há uma melhoria nas práticas parentais e nas relaçóes familiares, o que implica necessariamente práticas educativas parentais mais conscientes e presentes. ${ }^{(29)}$ Ademais, sendo a atenção plena nas práticas educativas parentais um modo de integrar pensamentos, sentimentos e comportamentos na esfera relacional com os filhos, reflete-se na forma como os pais adotam atitudes de compaixão, aceitação e bondade nas interaçóes que estabelecem - um processo gradual, sustentado numa série de ajustamentos e mudanças. ${ }^{(30)}$ Também, em contexto de saúde, os programas de educação parental que incluem a abordagem às técnicas de atenção plena têm vindo a impor-se. ${ }^{(14)}$

\section{Conclusão}

Os resultados deste estudo evidenciam melhoria nas competências parentais, diminuição de stress parental e aumento da atenção plena nas práticas educativas parentais, após participação no programa de educação parental ACT-RSK. Não obstante, salientamos algumas limitações. A amostra foi composta, maioritariamente, por mães (um pai em 22 participantes), colocando-se a questão do envolvimento paterno na educação parental. Ocorre ainda o facto de não haver grupo de controlo, bem como a ausência de um momento de follow-up. Não obstante os resultados relevantes nas competências parentais, stress parental e atenção plena nas práticas educativas parentais, é importante continuar a desenvolver estudos que procurem conhecer e explorar melhor de que modo esta relação se estabelece. Também o controlo da variável nível socioeconómico das famílias poderá ser importante. Sugerem-se estudos de caso que permitam abordagens específicas e em profundidade, bem como sustentar o investimento igualmente necessário em delineamentos de grupo e ensaios clínicos randomizados. A relevância dos efeitos da educação parental e, particularmente do programa ACT-RSK, depende muito, também, da preparação dos técnicos facilitado- 
res do programa e da sua acessibilidade aos pais. Neste contexto, estão em posição privilegiada os técnicos das áreas socioeducativa e da saúde, com particular relevo para os enfermeiros de família, pelo seu conhecimento profundo e proximidade das dinâmicas e problemáticas familiares de crianças e pais, podendo constituir-se o ACT-RSK como uma ferramenta fundamental na ação destes profissionais.

\section{Colaborações}

Santos AIPM, Martins ECF, Magalhães CCA, Mendes FED e Fernandes RIRS colaboraram com a concepção do projeto, coleta, análise e interpretação dos dados, análise e interpretação dos dados, redaçáo do artigo, revisão crítica relevante do conteúdo intelectual e aprovação final da versão a ser publicada.

\section{Referências}

1. Organização das Nações Unidas (ONU). Convenção sobre os Direitos da Criança. UNICEF; 1989.

2. Portugal. República Portuguesa. Decreto Abr 10, 1976. Aprova Constituição da República Portuguesa [internet]. [Citado 2020 Mar 14]. Disponível em: http://www.pgdlisboa.pt/leis/lei_mostra_articulado.php?nid=4\&tabela=leis

3. AltafimE, Pedro M, Linhares M. Effectiveness of ACT Raising Safe Kids Parenting Program in a developing country. Child Youth Serv Rev. 2016;70:315-23.

4. Sigmarsdótti M, Rains L, Forgatch M. Parent management training - Oregon Model: A Program to Treat Children`s Behavior. In: James JR. Evidence - Based Parenting Education: A Global Perspective: Routtledge: New York; 2016.

5. Kumpfer K, Magalhães C. Strengthening Families Program: an evidence-based family intervention for parents of high-risk children and adolescents. J Child Adolesc Subst Abuse. 2018;27(3):174-9.

6. Moreira H, Canavarro M. Psychometric properties of the interpersonal mindfulness in parenting scale in a sample of portuguese mothers. New York: Springer Science+Business Media; 2016.

7. Kumpfer K, Magalhães C, Kanse S. Impact of family structure, functioning, culture, and family-based interventions on children's health. In: Korin MR. editor. Health promotion for children and adolescents. New York: Springer. 2016; p.57-84.

8. Novaes M. Educação parental e prevenção intersetorial em saúde pública: A promoção e desenvolvimento da parentalidade positiva no Brasil. Coimbra: Universidade de Coimbra; 2015.

9. Liyana Amin NA, Tam WW, Shorey S. Enhancing first-time parents' selfefficacy: A systematic review and meta-analysis of universal parent education interventions' efficacy. Int J Nurs Stud. 2018;82:149-62.

10. Schmidt $B$, Staudt $A$, Wagner $A$. Intervenções para promoção de práticas parentais positivas: uma revisão integrativa. Contextos Clín. 2016;9(1):1-18.

11. Lopes N, Ferreira R. Intervenção dos enfermeiros na capacitação parental no relacionamento pais-filhos. Rev UIIPS. 2015;3(5):312-29.
12. Lopes MS Catarino H, Dixe M. Parentalidade positiva e enfermagem: Revisão sistemática da literatura. Rev Enf Ref. 2010;3 (1):109-18.

13. Gilmer C, Buchan JL, Letourneau N, Bennett CT, Shanker SG, FenwickA, et al. Parent education interventions designed to support the transition to parenthood: A realist review. Int J Nurs Stud. 2016;59:118-33.

14. Shorey S, Ang L, Chee CY. A systematic mixed-studies review on mindfulness-based childbirth education programs and maternal outcomes. Nurs Outlook. 2019;67(6):696-706.

15. American Psychological Association (APA). ACT Program for Parents: A snapshot [Internet]. Wahshingto (D.C.): APA; c2020. [cited 2020 Mar 24]. Available from: https://www.apa.org/act/parents

16. Knox M, Burkhart K. A multi-site study of the ACT Raising Safe Kids Program: predictors of outcomes and attrition. Child Youth Serv Rev. 2014;39:20-4.

17. Pedro M, Altafim E, Linhares M. ACT Raising Safe Kids Program to promote positive maternal parenting practices in different socioeconomic contexts. Interv Psicosoc. 2017;26(2):63-72.

18. Simões S. Formação parental: Implementação do programa ACT num grupo de pais da Região Centro de Portugal. Viseu: Instituto Politécnico de Viseu; 2018.

19. Macedo DM, Foschiera LN, Bordini TC, Habigzang LF, Koller SH. Revisão sistemática de estudos sobre registros de violência contra crianças e adolescentes no Brasil. Cien Saude Colet. 2019;24(2):487-96.

20. Altafim E, Mccoy D, Linhares M. Relations between parenting practices, socioeconomic status, and child behavior in Brazil. Child Youth Serv Rev. 2018;89:93-102.

21. Santos S. Adaptação portuguesa, para crianças em idade escolar, do Parenting Stress Index (PSI): resultados preliminares. Ver Port Psicol. 1992;28:115-32.

22. Santos S. Índice de Stress Parental (PSI). In: Leandro A, Simões M, Machado C, Gonçalves M, editors. Avaliação psicológica: Instrumentos validados para a população portuguesa. 2a ed. Coimbra: Quarteto; 2008. p. 123-34.

23. Brito A, Faro A. Estresse parental: revisão sistemática de estudos empíricos. Psicol Pesqui. 2016;10(1):64-75.

24. Leça ML. Estudo qualitativo sobre o Programa ACT: Exploração das perceções de pais de diferentes níveis socioeconómicos [dissertação]. Lisboa: Universidade de Lisboa; 2018.

25. Oliveira F. Intervenção preventiva em práticas educativas parentais aplicada a mães de crianças nascidas pré-termo na primeira infância [dissertação]. São Paulo: Universidade de São Paulo; 2018.

26. Silva J, Williams L. Um estudo de caso com o Programa Parental ACT para educar crianças em ambientes seguros. Trends Psychol. 2016;24(2):743-55.

27. Knox M, Burkhart K, Howe T. Effects of the ACT Raising Safe Kids Parenting Program on children's externalizing problems. Fam Relat. 2011;60(4):491-503.

28. Murray J, Santos IS, Bertoldi AD, Murray L, Arteche A, Tovo-Rodrigues $L$, et al. The effects of two early parenting interventions on child aggression and risk for violence in Brazil (The PIÁ Trial): protocol for a randomised controlled trial. Trials. 2019;20(1):253.

30. Ryzin M, Fosco G. Family - Based approaches to prevention: The State of the field. In: Ryzin M, Kumpfer K. Fosco G, Greeberg M, editors. Family - Based prevention Program for children and adolescent: theory research, and large - scale dissemination. New York: Taylor \& Francis Group; 2016. p. 1-20.

29. Coatsworth JD, Duncan LG, Greenberg MT, Nix RL. Changing parent's mindfulness, child management skills and relationship quality with their youth: results from a randomized pilot intervention trial. J Child Fam Stud. 2010;19(2):203-17. 